\title{
An Embedded Environmental Control Micro-chamber System for RRAM Memristor Characterisation
}

\author{
T. Abbey ${ }^{*}$, A. Serb ${ }^{* \dagger}$, N. Vasilakis ${ }^{*}$, L. Michalas ${ }^{*}$, A. Khiat ${ }^{*}$, S. Stathopoulos ${ }^{* \dagger}$ and T. Prodromakis ${ }^{* \dagger}$ \\ ${ }^{\dagger}$ ArC Instruments Ltd. 75 Sirocco, 33 Channel Way, Southampton, SO14 3JF, UK \\ *Nanoelectronics and Nanotechnology Research Group, Electronics and Computer Science Department, \\ University of Southampton, Southampton, SO17 1BJ, UK \\ ta5g14@soton.ac.uk
}

\begin{abstract}
Environmental conditions can greatly affect the performance of semiconductor devices. Great sophistication has thus gone into developing versatile systems that allow benchmarking of operating characteristics under a variety of temperature and humidity conditions. Recently, Resistive Random Access Memory (RRAM) technologies, also known as memristors, have received a lot of attention for memory and computing applications. This interest is showcased by several reports on technology and applications developments, as well as developments on the underpinning infrastructure, i.e. models and characterization tools, that renders such technologies useful. Several international research groups and companies are nowadays using ArC One ${ }^{\mathrm{TM}}$, a versatile instrument that allows en masse characterization of RRAM technologies, as has been presented previously in several demo sessions at ISCAS. In this work, we present a newly developed module that expands ArC One $^{\mathrm{TM}}$ capabilities through incorporating an environmental control system. The proposed module condenses the functionality of significantly larger, more complex and higher cost systems into a low cost, small form-factor and user friendly desktop-operated device. The system allows for temperature, atmospheric composition and humidity control and can be used for studying the impact of such settings on the electrical characteristics of RRAM technologies.
\end{abstract}

Keywords-RRAM; memristors; temperature; humidity; Environmental Control

\section{INTRODUCTION}

When characterising the behavior of a semiconductor device, external conditions such as temperature, humidity and atmospheric composition can significantly affect the performance of the device under test (DUT). Thorough characterisation of new technologies and/or investigation into device durability, necessitate the use of systems that allow controlling these parameters in a user-friendly manner. In existing systems, a probe station with a temperature-controlled chuck can be used to set the temperature of the DUT. This, however, involves a large and complex system that requires the user to manually align and connect delicate probes to the device's typically delicate test pads; notwithstanding the overall cost of such systems that often prohibits broad use.

Our group has previously developed an automated embedded characterization set-up [1], known as $\mathrm{ArC} O \mathrm{One}^{\mathrm{TM}}$, that allows the en masse characterization of RRAM memristors. The system presented in this paper extends the

This work was supported by the EPSRC grant. EP/K017829/1 and ArC Instruments ltd. capabilities of $\mathrm{ArC} \mathrm{One}^{\mathrm{TM}}$ by incorporating an environmental control system atop of it. The proposed system requires little experience and setup time, comprising only three connections between the micro-chamber itself and the control manifold, (one ribbon cable for electrical connections and two pneumatic connections). The designed system can currently control atmospheric humidity up to $50 \%$ above ambient, with a $\pm 1.5 \%$ error through use of a pneumatic pump and a manually developed bubbler, and ramp temperature up to $22 \mathrm{C}$ above ambient with better than $\pm 1 \%$ stability (proportional error in deg. C). The operating range for temperature is presently being extended up to a maximum temperature of $85 \mathrm{C}$ to allow for more extended studies on the thermal ageing of devices, rendering the system useful for both research and industrial applications. Overall, this system allows for rapid and accurate control of both temperature and humidity in a desktop system without the need for bulky and expensive hardware or specialist training.

The rapid memristor characterisation capabilities of the ArC One ${ }^{\mathrm{TM}}$ have been extensively demonstrated previously at ISCAS in 2014, 2016 and 2017 [2-4]. This work will thus focus on combining electrical characterisation of metal-oxide memristor devices with environmental condition control. The proposed system will thus provide added benefits to RRAM research and development for optimizing device performance as well as testing device robustness. Section II of this paper presents the system overview, with Section III describing the system's operation. Finally, the system is employed in Section IV for acquiring temperature and humidity dependent measurements of an in-house RRAM technology based on $\mathrm{TiO}_{\mathrm{x}}$ cells.

\section{SYSTEM OVERVIEW}

The environmental control system is divided into three main parts: the micro-chamber, the manifold construction and software. These systems are kept modular in order to allow for user configurability. For instance, if only the temperature control system is required, the user does not have to make the pneumatic attachments between the manifold and the microchamber, as depicted in Fig. 1.

\section{A. Micro-chamber}

The micro-chamber itself is constructed from 3 parts shown in Fig. 2: a printed circuit board (PCB) that forms the roof of the chamber and holds the environmental sensor and heating 


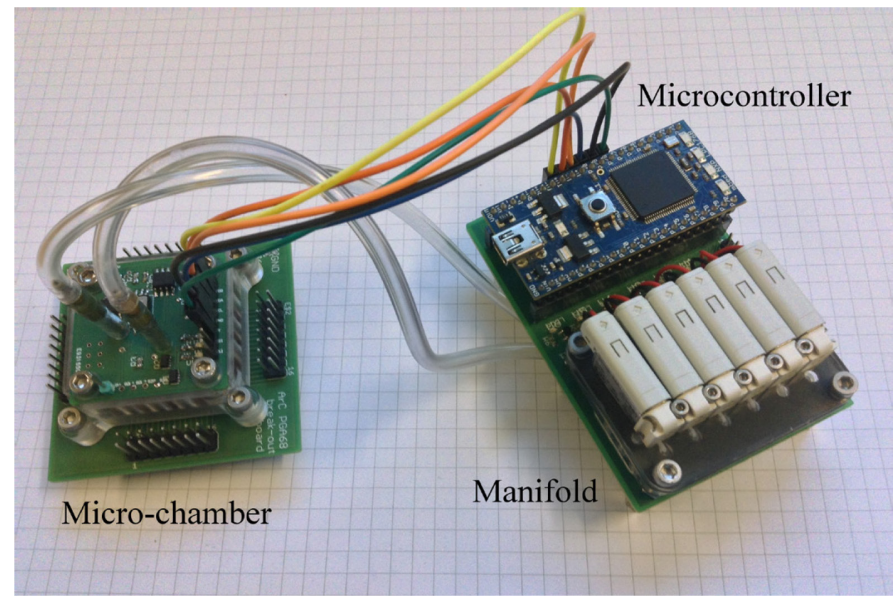

Fig. 1. The full environmental control system, showing the microchamber and manifold. Grid is $5 \mathrm{~mm}$ for all images.

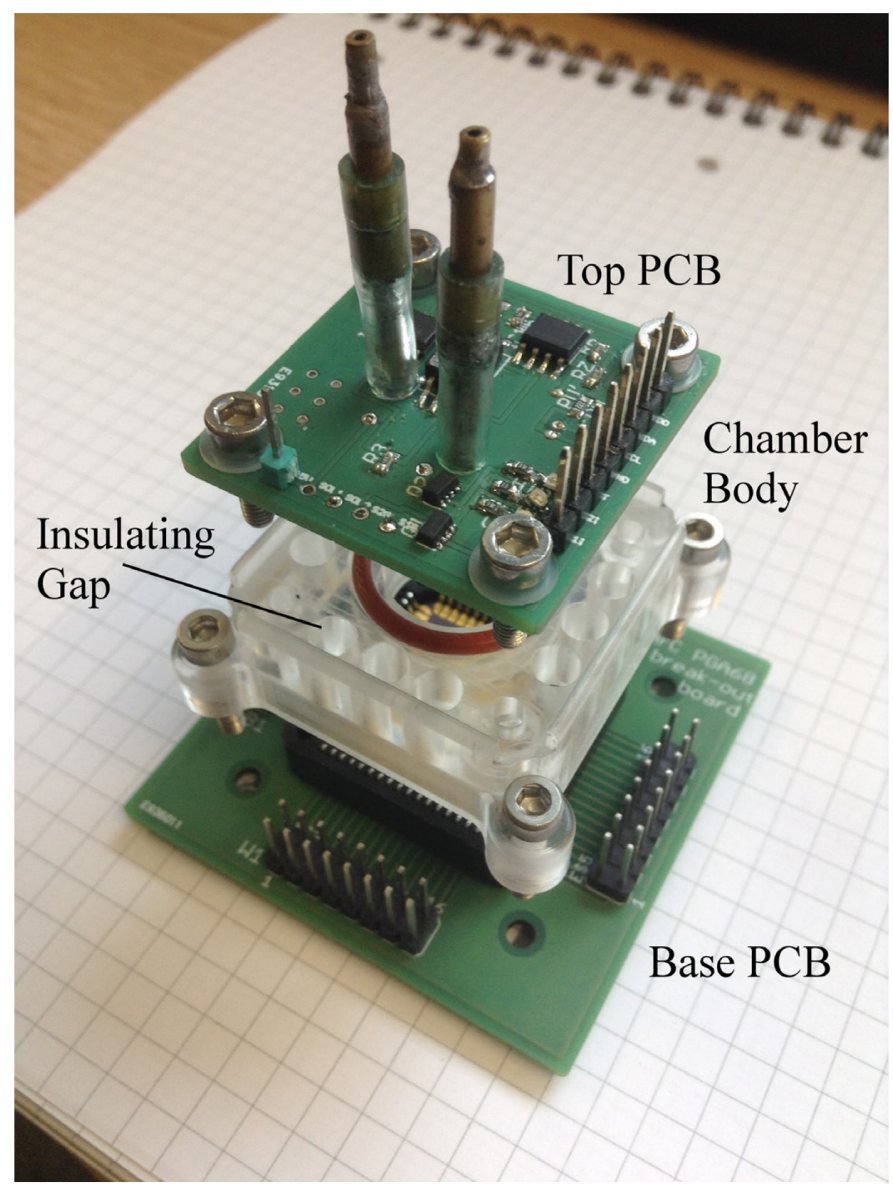

Fig. 2. Micro-chamber assembly blow-up, showing the separate PCB, acrylic body and base PCB. This version of the system is missing the insulating rubber layer underneath the PCB. The insulating gaps built into the chamber body can be seen.

elements, the body of the chamber and the removable base in which the DUT is mounted. The body of the micro-chamber is manufactured from acrylic that seals with the PLCC68 packaged platform. Thermal losses through the walls of the system were minimized by including air gaps in the chamber walls, a rubber sheet to insulate the top PCB from the inside of the micro-chamber and nylon washers between the securing

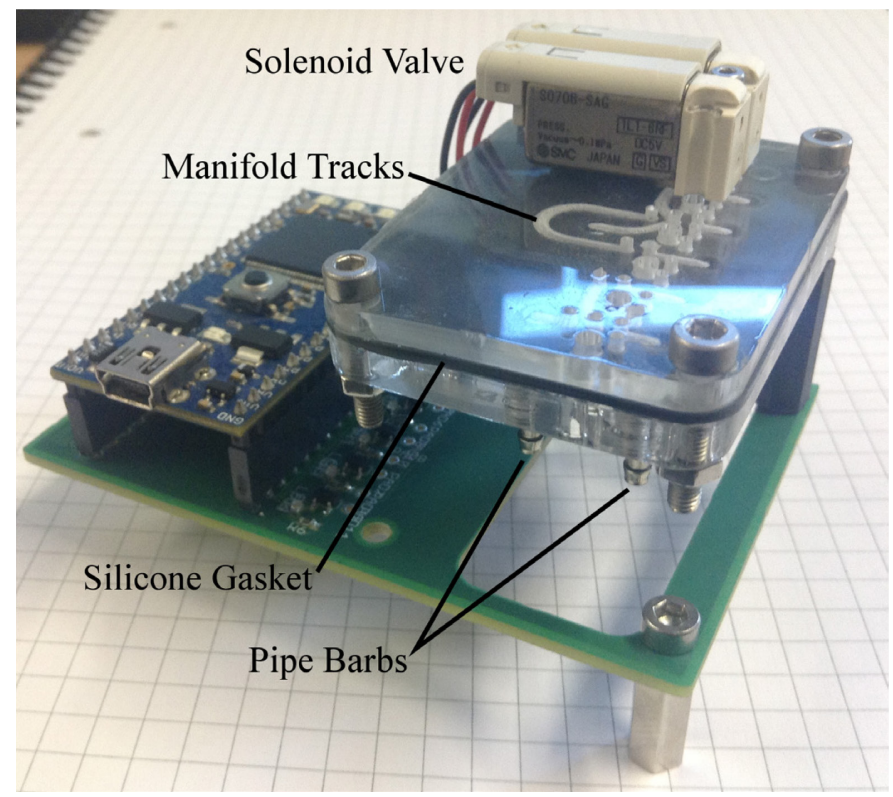

Fig. 3. The manifold assembly showing the manifold construct and connection interfaces. This version of the system has three solenoid valves that were made redundant in later iterations.

points of the PCB to minimize conductive losses

The base PCB hosts the PLCC68 package holder and provides the headers for interfacing with the $\mathrm{ArC} \mathrm{One}{ }^{\mathrm{TM}}$. The package in the holder forms the base of the micro-chamber through use of a silicone o-ring pressing against the package, secured by the four corner screws on the body. In summary, the top PCB provides the environmental sensing and control, and the base PCB provides electrical connections to the DUT.

\section{B. Manifold}

Similarly to the micro-chamber, the manifold is a multi-part construction as depicted in Fig. 3. The gas control of the system is achieved with three solenoid valves, which sit on top of a PMMA-Silicone-PMMA sandwich structure. Pneumatic channels directing gas flow are laser-etched on the top PMMA layer and kept air-tight by the Silicone gasket. The bottom PMMA plate offers mechanical support and firmly fixes the gasket against the top PMMA plate.

The manifold features two inlet valves, which can be independently controlled by the user. This allows for control of atmospheric composition inside the chamber simply by connecting two different gas sources. The manifold structure itself is mounted atop a PCB hosting the microcontroller operating the system and necessary switching components, thus minimising external connections.

\section{Software}

Both the manifold and micro-chamber are controlled using a central microcontroller, the mbed NXP LPC1768 hosted by the manifold PCB. This, in turn, connects to a host computer through with the control parameters are entered using a terminal software such as Teraterm. The control scheme for the parameters of the system is achieved using simple proportional integral derivative (PID) control. The gains for these control systems were calibrated manually by observing the system 
response to input values. Temperature and humidity control systems operate independently, allowing for simultaneous or individual operation at no loss of accuracy.

\section{SYSTEM TESTING AND CALIBRATION}

The system was tested and manual calibrations of the control systems were undertaken.

\section{A. Temperature Control}

The temperature control shown in Fig. 4 is achieved in this system through the use of resistors, totaling $1.2 \mathrm{~W}$ output power. Calculations using Fourier's law of thermal conduction, equation (2), indicate a theoretical maximum temperature increase of $25.06 \mathrm{C}$, whereas practical measurements give a result of $22.32 \mathrm{C}$, a $10.9 \%$ discrepancy.

$$
\frac{q}{A}=\frac{k \Delta T}{s}
$$

$\mathrm{q}$ is the heat flux (at equilibrium the total heat flux is the power of the heater), $\mathrm{A}$ is the surface area of the conducting surface under consideration, $\mathrm{k}$ is its corresponding thermal conductivity, $\mathrm{s}$ is the thickness of the conductive surface and $\Delta \mathrm{T}$ is the temperature difference between the inside and outside of the micro-chamber. Applying this equation to the microchamber body, the top PCB and the ceramic package delimiting the micro-chamber from the bottom side, the $25.06 \mathrm{C}$ approximation is arrived at.

\section{B. Humidity Control}

If the system is equipped with a bubbler and external pump, humidity control is also possible through toggling of the inputs, one input being for dry air and one for air passed through the bubbler. This system allows for humidity control between ambient and a $50 \%$ increase $(35-85 \% \mathrm{RH})$ when both the chamber and water are at room temperature $(22 \mathrm{C})$. The humidity can be increased further through the use of hot water in the bubbler, this however impacts the temperature control of the system. The temperature control and humidity control can be used in conjunction. This reduces the maximum achievable humidity to a $35 \%$ increase for a temperature increase of around $10 \mathrm{C}$.

The same infrastructure can be used to control the gas mix in the chamber instead by attaching different gas sources to the inputs. This would allow the user to for example, control the percentage of oxygen in the atmosphere of the device, or ensure the device is operated under an inert atmosphere. The response for the humidity control system is shown in Fig. 5.

\section{RESUlts}

The temperature control system has been demonstrated to be capable of reaching and controlling user defined temperatures up to $22 \mathrm{C}$ above ambient conditions with stability of better than $\pm 1 \% \Delta \mathrm{T}$ error (fig 4). The humidity control system has been demonstrated to be capable of achieving a $50 \%$ increase in the chamber humidity with a control error of $\pm 1.5 \%$ once stable, as demonstrated in Fig 5 . The PID

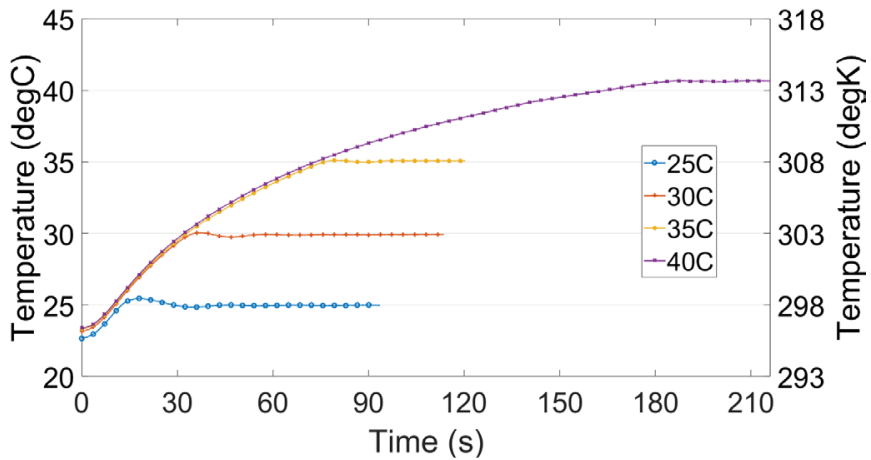

Fig. 4. Temperature response of the system for 25C, 30C, 35C and $40 \mathrm{C}$ showing classic PID controller characteristics. Room temperature is $22.6 \mathrm{C}$.

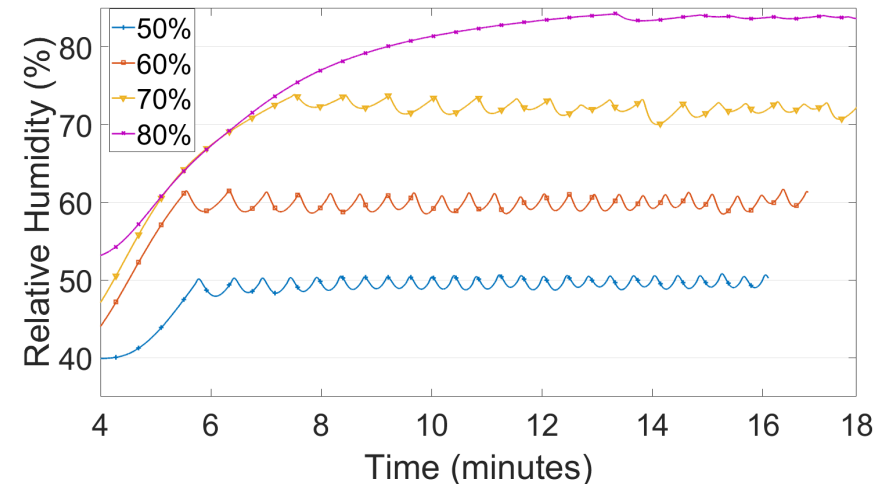

Fig. 5. The response of the humidity control system for $50 \%, 60 \%, 70 \%$ and $80 \%$ relative humidity. Room temperature was $24.2 \mathrm{C}$, room humidity was $36.8 \%$.

parameters for this system allow for better tuneability towards achieving a reduced settling time.

Considering our system's primary targeted application on RRAM characterization, a $\mathrm{Pt} / \mathrm{TiO}_{\mathrm{x}} / \mathrm{Pt}$ device was packaged in a PLCC68 holder and tested in the chamber. The current voltage (I/V) characteristics of these devices are typically determined by thermally activated conduction mechanisms and therefore by increasing the temperature the measured current is expected to consequently increase, offering a straightforward evaluation for the functionality of our setup.[5]

$\mathrm{I} / \mathrm{V}$ measurements were performed at $300 \mathrm{~K}, 310 \mathrm{~K}$ and $320 \mathrm{~K}$, sweeping the voltage in staircase mode between $[-1$, $+1] \mathrm{V}$ using the $\mathrm{ArC} \mathrm{One}^{\mathrm{TM}}$ (Fig. 6). The results are presented in Fig. 7. Measured current increases with higher temperature as expected, offering experimental confirmation of system functionality.

The effect of humidity was also evaluated. Oxide films deposited by sputtering techniques, such as those typically utilized in RRAMs, may absorb moisture due to their microstructure upon ambient exposure. The presence of moisture could furthermore significantly influence their performance [6-7]. Humidity testing was conducted by recording $\mathrm{I}-\mathrm{V}$ curves under different relative humidity conditions ranging from $35 \%$ to $65 \%$ at $30 \mathrm{C}$ as presented in Fig. 8. These conditions were achieved using the bubbler and pump setup. The tests showed increasing humidity causing an $8 \%$ reduction in conductivity at positive voltages, a $14 \%$ 


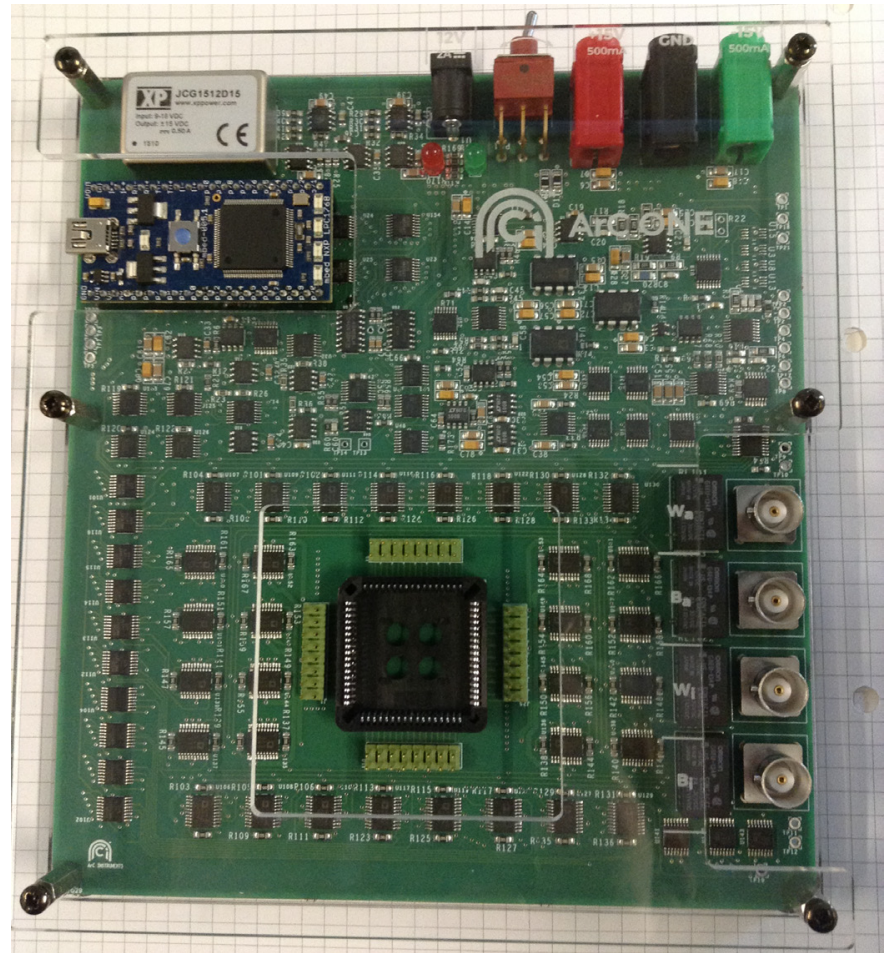

Fig. 6. The ArC One $\mathrm{TM}^{\mathrm{TM}}$ non-volatile memory device characterization system.

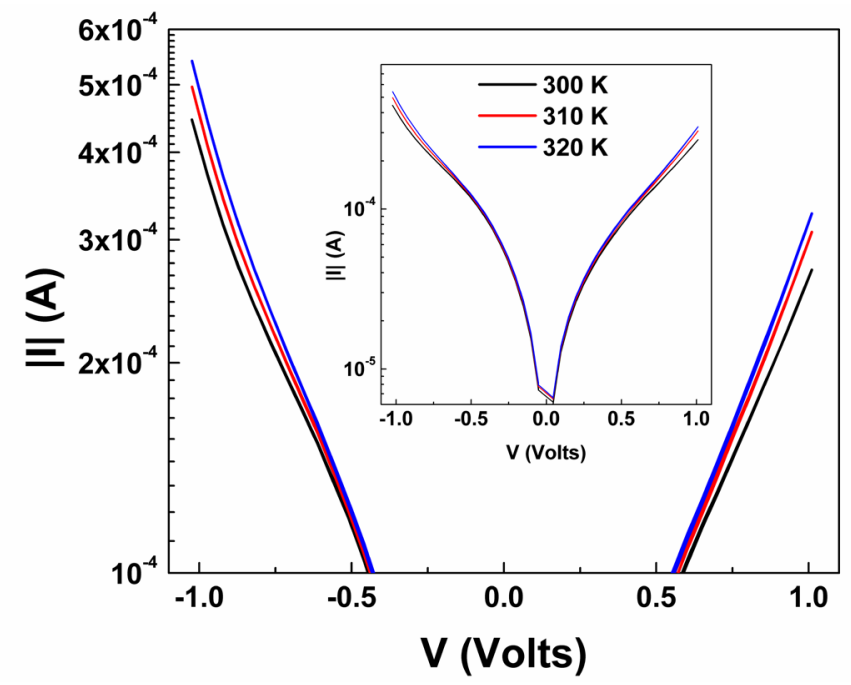

Fig. 7. The I/V curve for the packaged memristor at 300,310 and $320^{\circ} \mathrm{K}$. Temperature controlled using the micro-chamber.

increase in conductivity for negative voltages and an enhancement in the size of the hysteresis loops of the memristor.

\section{CONCLUSION}

In this work, we presented an embedded micro-chamber system that allows for the in-situ tuning of temperature and humidity in conjunction with a mobile memory characterization instrumentation platform. The designed system works to an accuracy of less than $1 \% \Delta \mathrm{T}$ error for temperature control and $1.5 \%$ error for humidity control, tuned

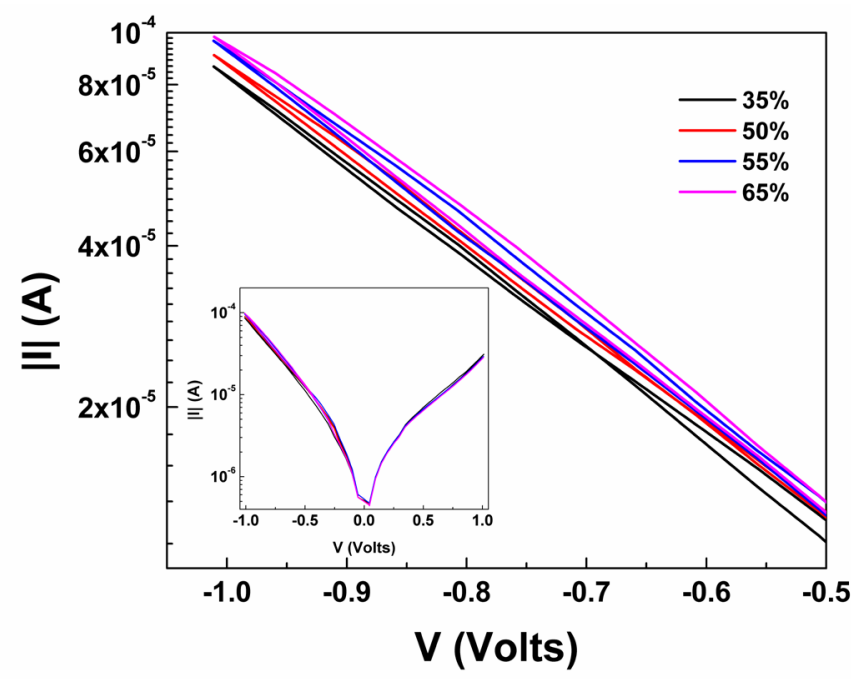

Fig. 8. The I/V curve for a memristor at $35 \%, 50 \%, 55 \%$ and $65 \%$ relative humidity at $30^{\circ} \mathrm{C}$.

through a highly compact, low cost and user friendly desktop system. This greatly reduces the user's requirement to use large, expensive and complex systems for external parameter control of devices inside the chamber. Our combined ArC one - micro-chamber setup has the capability to be used for assessing the impact of environmental parameters on device performance, investigate failure mechanisms and device reliability and explore potential environmental sensing abilities of memristive devices.

\section{REFERENCES}

[1] R. Berdan, A. Serb, A. Khiat, A. Regoutz, C. Papavassiliou and T. Prodromakis, "A $\mu$-Controller-Based System for Interfacing Selectorless RRAM Crossbar Arrays," in IEEE Transactions on Electron Devices, vol. 62, no. 7, pp. 2190-2196, July 2015.

[2] Serb, R. Berdan, A. Khiat, C. Papavassiliou and T. Prodromakis, "Live demonstration: A versatile, low-cost platform for testing large ReRAM cross-bar arrays," 2014 IEEE International Symposium on Circuits and Systems (ISCAS), Melbourne VIC, 2014, pp. 441-441.

[3] R. Berdan, A. Serb, A. Khiat, C. Papavassiliou and T. Prodromakis, "Live demonstration: Characterization of RRAM crossbar arrays at a click of a button," 2016 IEEE International Symposium on Circuits and Systems (ISCAS), Montreal, QC, 2016, pp. 1443-1443.

[4] I. Messaris et al., "Live demonstration: A TiO2 ReRAM parameter extraction method," 2017 IEEE International Symposium on Circuits and Systems (ISCAS), Baltimore, MD, 2017, pp. 1-1.

[5] S.M. Sze and K.K. Ng, Physics of Semiconductor Devices, John Wiley \& Sons 2006.

[6] T. Tsuruoka, K. Terabe, T. Hasegawa, I. Valov, R. Waser and M. Aono, "Effects of Moisture on the Switching Characteristics of Oxide-Based, Gapless-Type Atomic Switches." Advanced Functional Materials, vol. 22, no. 1, pp. 70-77, Jan. 2012.

[7] Q. Yin, C. Wei, Q. Wei, Y. Chen, Y. Xia, B. Xu, J. Yin and Z. Liu, "Polarity-dependent effect of humidity on the resistive switching characteristics of nonpolar devices.” Applied Physics Express, Vol. 9, no. 10, p.104202, Sep. 2016. 